\title{
Desafios para implementação da Sistematização da Assistência de Enfermagem na Terapia Intensiva: revisão integrativa
}

\author{
Challenges for implementing the Systematization of Nursing Care in Intensive Care: an integrative \\ review
}

Retos para la implementación de la Sistematización de la Atención de Enfermería en Cuidados

Intensivos: una revisión integradora

Recebido: 14/09/2021 | Revisado: 18/09/2021 | Aceito: 22/09/2021 | Publicado: 26/09/2021

Larissa dos Santos Silva
ORCID: https://orcid.org/0000-0001-8485-4094
Universidade Estadual do Piaú, Brasil
E-mail: 1ssilva@aluno.uespi.br
Ana Klara Rodrigues Alves
ORCID: https://orcid.org/0000-0002/1216-9386
Universidade Estadual do Piauí, Brasil
E-mail: klaraphb@outlook.com
Barbara Beatriz Lira da Silva
ORCID: https://orcid.org/0000-0001-9166-6147
Universidade Estadual do Piauí, Brasil
E-mail:brbeatriz16@gmail.com
Fábio Dias Nogueira
Faculdade de Ciências Humanas, Exatas e da Saúde do Piaú, Brasil
E-mail: fdnvaq@gmail.com
ORCID: https://orcid.org/0000-0001-7303-1180
Francisco Robson de Oliveira Alves
ORCID: https://orcid.org/0000-0002-9854-6460
Universidade Federal do Piaú, Brasil
E-mail: robson_oliveira.fisio@outlook.com
Iohana Santos de Vasconcelos
ORCID: https://orcid.org/0000-0002-7727-5622
Universidade Estadual do Piaú, Brasil
E-mail: iohana.vasconcelos007@gmail.com
Taynara Lais Silva
ORCID: https://orcid.org/0000-0003-1558-0506
Universidade Estadual do Piaú, Brasil
E-mail: taynaralaissilva@gmail.com
Senira de Oliveira Rodrigues Lavor
ORCID: https://orcid.org/0000-0002-5225-1034
Universidade Estadual do Piauí, Brasil
E-mail: seniraoliveira@phb.uespi.br

\section{Resumo}

A Sistematização da Assistência em enfermagem (SAE) é crucial à prestação de uma assistência de enfermagem segura, pois proporciona ao enfermeiro recursos técnicos, científicos e humanos, além de melhorar a qualidade de assistência prestada ao cliente. Dessa forma, a Unidade de Terapia Intensiva (UTI) é uma das unidades de cuidados mais complexas em uma instituição hospitalar. O presente estudo tem como objetivo analisar os desafios da equipe de enfermagem para a implementação da SAE nas UTIs. Trata-se de uma Revisão Integrativa. Para a busca das publicações foram selecionados os seguintes descritores: Unidade de terapia intensiva; Processo de Enfermagem; Serviços de Enfermagem, sendo utilizado o operador booleano AND para o cruzamento dos descritores. A busca foi realizada online no mês de janeiro de 2021, nas bases de dados CINAHL, MEDLINE, LILACS e BDENF. Após a aplicação dos critérios de inclusão e exclusão, obteve-se uma amostra final de 10 artigos. Foram elaboradas duas categorias temáticas: "Dificuldades vivenciadas por enfermeiros no processo de sistematização da assistência na UTI" e "Desafios relacionados à estruturação do processo na implementação da SAE nas UTIs". Assim foi identificado diversos desafios, como: falta de conhecimento teórico, número reduzido de profissionais; sobrecarga de trabalho, falta de tempo e má elaboração dos impressos. Dessa forma, a introdução de disciplinas que englobem a SAE nas graduações de enfermagem bem como maior envolvimento da instituição hospitalar contribuem para a conquista de benefícios próprios para a instituição e para os pacientes, por meio da implementação da SAE de forma competente e qualificada.

Palavras-chave: Unidade de terapia intensiva; Processo de enfermagem; Serviços de enfermagem. 


\begin{abstract}
The Systematization of Nursing Care (SAE) is crucial to providing safe nursing care, as it provides nurses with technical, scientific and human resources, in addition to improving the quality of care provided to the client. Thus, the Intensive Care Unit (ICU) is one of the most complex care units in a hospital. The present study aims to analyze the challenges of the nursing team for the implementation of SAE in ICUs. This is an Integrative Review. To search for publications, the following descriptors were selected: Intensive care unit; Nursing Process; Nursing Services, using the Boolean operator AND for the crossing of descriptors. The search was carried out online in January 2021, in the databases CINAHL, MEDLINE, LILACS e BDENF. After applying the inclusion and exclusion criteria, a final sample of 10 articles was obtained. Two thematic categories were elaborated: "Difficulties experienced by nurses in the process of systematizing care in the ICU" and "Challenges related to structuring the process in the implementation of SAE in the ICUs". Thus, several challenges were identified for the implementation of SAE in ICUs, such as: lack of theoretical knowledge, reduced number of professionals; overload of work, lack of time and poor preparation of the forms. Thus, the introduction of disciplines that include SAE in nursing degrees as well as greater involvement of the hospital institution contribute to the achievement of benefits for the institution and for patients, through the implementation of SAE in a competent and qualified manner.
\end{abstract}

Keywords: Intensive care unit; Nursing process; Nursing services.

\title{
Resumen
}

La Sistematización de la Atención de Enfermería (SAE) es fundamental para la prestación de una atención de enfermería segura, ya que brinda al enfermero recursos técnicos, científicos y humanos, además de mejorar la calidad de la atención brindada al cliente. Así, la Unidad de Cuidados Intensivos (UCI) es una de las unidades de cuidados más complejas de una institución hospitalaria. Este estudio tiene como objetivo analizar los desafíos que enfrenta el equipo de enfermería en la implementación de SAE en UCI. Es una revisión integradora. Para la búsqueda de publicaciones se seleccionaron los siguientes descriptores: unidad de cuidados intensivos; Proceso de enfermería; Servicios de enfermería, utilizando el operador booleano Y para el cruce de descriptores. La búsqueda se realizó online en enero de 2021, en las bases de datos CINAHL, MEDLINE, LILACS y BDENF. Tras aplicar los criterios de inclusión y exclusión, se obtuvo una muestra final de 10 artículos. Se elaboraron dos categorías temáticas: "Dificultades vividas por enfermeras en el proceso de sistematización de la atención en la UCI" y "Desafíos relacionados con la estructuración del proceso en la implementación de SAE en las UCI". Así, se identificaron varios desafíos, tales como: falta de conocimientos teóricos, número reducido de profesionales; sobrecarga de trabajo, falta de tiempo y mala preparación del material impreso. Así, la implantación de disciplinas que engloban al SAE en las titulaciones de enfermería, así como una mayor implicación de la institución hospitalaria, contribuyen al logro de beneficios para la institución y para los pacientes, a través de la implementación del SAE de manera competente y calificada.

Palabras clave: Unidad de cuidados intensivos; Proceso de enfermería; Servicios de enfermería.

\section{Introdução}

O reconhecimento da enfermagem como ciência é inegável, porém é por meio da comprovação e divulgação de evidências científicas que se enaltece a enfermagem como ciência capaz de transformar a realidade do local no qual está inserida. Além disso, a organização da assistência de enfermagem, com o objetivo de garantir segurança aos pacientes e de melhorar as condições de trabalho é a base principal para o fortalecimento da enfermagem como profissão (Abreu, 2020).

No que tange aos aspectos legais, a Resolução no 272/2002 foi revogada pela Resolução 358/2009, do COFEN, que dispõe sobre a SAE e a implementação do Processo de Enfermagem (PE) em ambientes públicos ou privados, em que ocorre o cuidado de enfermagem. É uma atividade privativa do enfermeiro, utiliza métodos e estratégia de trabalho científico para a identificação das situações de saúde/doença, subsidiando ações de assistência de Enfermagem que possam contribuir para a promoção, prevenção, recuperação e reabilitação da saúde do indivíduo, família e comunidade (Nery, Santos \& Sampaio, 2013).

Desta forma, o uso da SAE é crucial à prestação de uma assistência de enfermagem segura, pois proporciona ao enfermeiro recursos técnicos, científicos e humanos, melhora a qualidade de assistência prestada ao cliente, e possibilita o reconhecimento e a valorização da enfermagem frente à sociedade (Gutierres, et al., 2018).

De acordo com Silva, et al. (2017), a SAE é compreendida como todo conteúdo/ação que organize o trabalho profissional do enfermeiro, com base teórico-filosófica, que possibilite a operacionalização do PE, com base teórico-filosófica.

Conforme Swiger, et al. (2016), o cuidado sistematizado imprime qualidade aos resultados, pois permite que a ação de ajuda que o paciente precisa receber seja particularizada, traduzindo a melhor maneira de implementação. Desta forma quando 
desenvolvido com competência, têm o objetivo de favorecer a mobilização das potencialidades das pessoas, no sentido de prestar atendimento digno, sensível, e resolutivo para promover, prevenir e recuperar. E frente a impossibilidade de cura, proporcionar uma morte digna minimizando o sofrimento humano.

A Unidade de Terapia Intensiva (UTI) é uma das unidades de cuidados mais complexas em uma instituição hospitalar. Neste setor ficam internados os clientes com quadros clínicos e cirúrgicos mais complexos que necessitam de equipamentos de alta precisão para a detecção de anormalidades o mais rápido possível, objetivando em um diagnóstico imediato e um cuidado organizado e mais completo (Viana, et al., 2018).

O PE é essencial para que haja uma assistência de qualidade onde ocorre o cuidado profissional de Enfermagem. Em uma Unidade de Terapia Intensiva, lugar onde estão os pacientes mais críticos, esse cuidado sistematizado é imprescindível para uma assistência efetiva e de excelência, contribuindo positivamente para o cuidado, pois intensifica a organização e estruturação do setor (Viana, et al., 2018).

Dessa forma, o artigo tem como objetivo conhecer os desafios para a implementação da Sistematização da Assistência de Enfermagem em Unidades de Terapia Intensiva, de acordo com as produções científicas.

\section{Metodologia}

Trata-se de uma Revisão Integrativa da Literatura (RIL). A RIL é um tipo de estudo que foi desenvolvida pelo advento da pesquisa baseada em evidência e objetiva analisar pesquisas relevantes e extrair as principais conclusões para subsidiar a tomada de decisão do enfermeiro, além de, indicar lacunas do conhecimento que precisam ser pesquisadas para serem respondidas. Mendes \& Silveira \& Galvão (2008). Nesta pesquisa foram seguidas as seis fases da elaboração da revisão integrativa: elaboração da pergunta norteadora, busca ou amostragem na literatura, coleta de dados, análise crítica dos estudos incluídos, discussão dos resultados e apresentação da revisão integrativa.

Na primeira etapa foi elaborada como questão norteadora: Quais os desafios para a implementação da Sistematização da Assistência de Enfermagem em Unidades de Terapia Intensiva, de acordo com as produções científicas?

Após estabelecer a pergunta norteadora, como fase fundamental na construção da revisão integrativa, definiu-se os descritores para conduzir a pesquisa nas bases de dados bem como o operador booleano. Foram eleitos os seguintes descritores encontrados no DeCS (Descritores em Ciências da Saúde) e seus correspondentes na língua inglesa que compõem o Medical Subject Headings (MESH): "Unidade de terapia intensiva” (Intensive Care Unit); "Processo de Enfermagem” (Nursing Process); "Serviços de Enfermagem" (Nursing Services), sendo utilizado o operador booleano AND para o cruzamento desses descritores.

Com vistas à identificação das publicações que comporiam a amostra desta revisão, foram realizadas buscas online no mês de janeiro de 2021 nas bases de dados da Cinahl Information Systems (CINAHL), US National Library of Medicine (MEDLINE) via PubMed; Literatura Latino-americana e do Caribe em Ciências da Saúde (LILACS) e Base de dados em Enfermagem (BDENF).

Para seleção das publicações foram adotados os seguintes critérios de inclusão: artigos completos, disponíveis gratuitamente na íntegra, publicados em português, inglês e espanhol nos últimos cinco anos (2016 - 2021) e que retratassem a temática referente, com vistas a obter as pesquisas mais relevantes e recentes sobre a temática. Para a seleção dos manuscritos, dois pesquisadores independentes avaliaram os resumos dos estudos previamente identificados. Quando havia dúvidas ou discordâncias, um terceiro pesquisador era consultado para decidir sobre a inclusão ou não da publicação na amostra. Foram excluídos os artigos que não se enquadravam nos critérios de inclusão, bem como aqueles que estavam repetidos nas duas bases de dados pesquisadas e os manuscritos que não respondiam aos objetivos propostos para este estudo, após a leitura preliminar dos seus resumos.

Foram encontrados na base de dados CINAHL um total de 92 artigos, na base Medline via PubMed foram identificados 
165 manuscritos, na Lilacs foram encontrados um total de 67 artigos e na BDENF foram identificados 55 manuscritos, totalizando 379 publicações. Após a aplicação dos critérios de inclusão e exclusão, obteve-se uma amostra final de 10 artigos, dos quais um pertencia à base de dados CINAHL, dois ao Medline, três ao Lilacs e quatro na BDENF. A Figura 1 mostra o fluxograma do processo de seleção dos manuscritos.

Figura 1 - Fluxograma explicativo do processo de seleção dos manuscritos. Parnaíba, Piauí, Brasil- 2021.

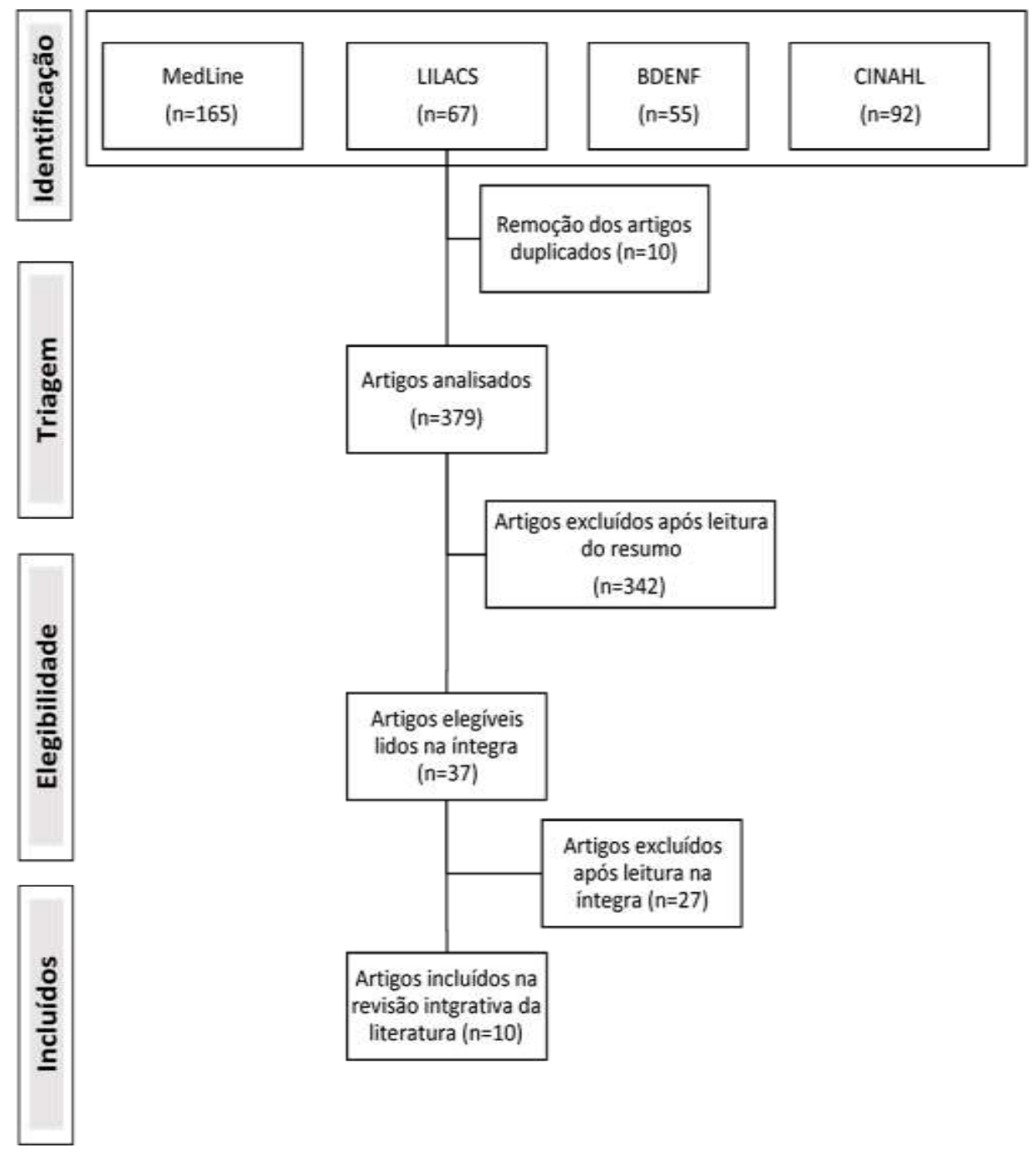

Fonte: Elaboração própria (2021).

Para extrair as informações mais relevantes dos artigos selecionados, utilizou-se instrumento de coleta de dados validado por Ursi. Após a análise crítica das investigações e o preenchimento do referido instrumento, os resultados de cada estudo que respondiam à questão norteadora foram inseridos em um quadro. Posteriormente, foi realizada a discussão dos resultados a partir de categorias temáticas e a apresentação da revisão integrativa. Por se tratar de estudo de revisão, no qual não há envolvimento com seres humanos, este estudo não foi encaminhado para a apreciação do Comitê de Ética em Pesquisa, conforme Resolução 510/16 (Brasil, 2016), porém foram reconhecidos os aspectos éticos de uma revisão integrativa, sendo respeitadas as autorias de todas as fontes que foram citadas nesta pesquisa. 


\section{Resultados e Discussão}

Para caracterização dos artigos, foi elaborado um quadro com os seguintes itens: ordem, título; ano/ base de indexação/periódico; autores; tipo de estudo e nível de evidência, conforme mostra o Quadro 1.

Os 10 artigos que compuseram a amostra final do estudo receberam uma identificação alfanumérica de A1 a A10, de acordo com a ordem em que foram analisados e identificados no instrumento de coleta de dados validado por Ursi.

Neto e Schuilze (2015) classificam a hierarquia entre as evidências, dependendo do tipo de estudo, sendo a hierarquia da melhor evidência para a evidência mais frágil, ou seja, do Nível I para o Nível VII.

Quadro 1 - Caracterização dos estudos incluídos na revisão integrativa acerca do tema Análise da implantação da Sistematização da Assistência de Enfermagem em unidades de terapia intensiva segundo a ordem, título do manuscrito, ano/base de indexação/periódico, autores, tipo de estudo, síntese dos resultados encontrados e nível de evidência. Parnaíba, Piauí, Brasil2021.

\begin{tabular}{|c|c|c|c|c|c|c|}
\hline Ordem & Título & $\begin{array}{l}\text { Ano/Base de } \\
\text { indexação/ } \\
\text { Periódico }\end{array}$ & Autores & $\begin{array}{l}\text { Tipo de } \\
\text { Estudo }\end{array}$ & Síntese dos resultados encontrados & $\begin{array}{l}\text { Nível de } \\
\text { Evidência }\end{array}$ \\
\hline A1 & $\begin{array}{lr}\text { Percepções } & \text { dos } \\
\text { enfermeiros } & \text { acerca } \\
\text { da implementação } \\
\text { do processo } r \\
\text { enfermagem } \text { em } \\
\text { uma } \\
\text { intensiva }\end{array}$ & $\begin{array}{lr}2021 / & \text { BDENF/ } \\
\text { Revista Gaúcha } \\
\text { Enfermagem }\end{array}$ & $\begin{array}{l}\text { Alexsandra Martins da } \\
\text { Silva; Aline Daiane } \\
\text { Colaço; Camila Vicente; } \\
\text { Kátia Cilene Godinho } \\
\text { Bertoncello; } \quad \text { Lúcia } \\
\text { Nazareth Amante; } \\
\text { Mabel Villa Demetrio }\end{array}$ & Descritivo & $\begin{array}{l}\text {-Desinteresse, resistência e } \\
\text { desvalorização do método } \\
\text { - Número reduzido de profissionais } \\
\text {-Processo tro de trabalho } \\
\text { individualizado/falha no trabalho } \\
\text { - Sobrecarga de trabalho } \\
\text {-Necessidade de capacitações e } \\
\text { atualizações }\end{array}$ & VI \\
\hline A2 & $\begin{array}{lr}\text { Boas Práticas } & \text { de } \\
\text { Enfermagem ra } & \text { na } \\
\text { Unidade de Terapia } \\
\text { Intensiva: } \\
\text { Desenvolvimento o } \\
\text { histórico r de } \\
\text { enfermagem. }\end{array}$ & $\begin{array}{l}2020 / \quad \text { LILACS/ } \\
\text { Revista } \\
\text { Enfermagem em } \\
\text { Foco }\end{array}$ & $\begin{array}{lr}\text { Marisa Gomes dos } \\
\text { Santos; Tatiana Gaffuri } \\
\text { da Silva; Alexsandra } \\
\text { Martins da Silva; Júlia } \\
\text { Valéria de Oliveira } \\
\text { Vargas ratencourt; } \\
\text { Eliane Regina Pereira do } \\
\text { Nascimento; r Katia } \\
\text { Cilene r Godinho } \\
\text { Bertoncello }\end{array}$ & Qualitativo & $\begin{array}{l}\text { - Divergência entre raciocínio e } \\
\text { prática } \\
\text { - Falta de conhecimento teórico. } \\
\text { - A tecnologia do cuidado que orienta } \\
\text { o raciocínio e melhora a qualidade do } \\
\text { cuidado. } \\
\text { - Necessidade de capacitações e } \\
\text { atualizações }\end{array}$ & VI \\
\hline A3 & $\begin{array}{lr}\text { Significados } & \text { de } \\
\text { sistematização } & \text { da } \\
\text { assistência } & \text { de } \\
\text { enfermagem sob } & \text { a } \\
\text { ótica de enfermeiros }\end{array}$ & $\begin{array}{l}\text { 2020/CINAHL/ } \\
\text { Revista Práxis }\end{array}$ & $\begin{array}{l}\text { Ana Caroline da Costa; } \\
\text { José Vitor da Silva }\end{array}$ & Descritivo & $\begin{array}{l}\text {-Contribuem para melhoria do } \\
\text { serviço. } \\
\text {-Aumenta vínculo } \\
\text { profissional/paciente. } \\
\text {-Sobrecarga de trabalho. } \\
\text { - Divergência entre raciocínio e } \\
\text { prática } \\
\text { - Falta de conhecimento teórico. } \\
\text {-Necessidade de capacitações e } \\
\text { atualizações }\end{array}$ & VI \\
\hline A4 & $\begin{array}{lr}\text { Documentação } & \text { do } \\
\text { processo } & \text { de } \\
\text { enfermagem } & \text { em }\end{array}$ & $\begin{array}{lr}\text { 2019/ MEDLINE/ } \\
\text { Revista da Escola }\end{array}$ & $\begin{array}{l}\text { Oswalcir Almeida de } \\
\text { Azevedo; Érika de } \\
\text { Souza Guedes; Sandra }\end{array}$ & Descritivo & $\begin{array}{l}\text { - O setor que mais documentavam no } \\
\text { hospital era as unidades de terapia } \\
\text { intensiva. }\end{array}$ & VI \\
\hline
\end{tabular}




\begin{tabular}{|c|c|c|c|c|c|c|}
\hline & $\begin{array}{l}\text { instituições } \\
\text { públicas de saúde }\end{array}$ & $\begin{array}{l}\text { em Enfermagem da } \\
\text { USP }\end{array}$ & $\begin{array}{llr}\text { Alves } & \text { Neves } & \text { Araújo; } \\
\text { Magda } & \text { Maria } & \text { Maia; } \\
\text { Diná de } & \text { Almeida Lopes } \\
\text { Monteiro da Cruz }\end{array}$ & & $\begin{array}{l}\text { - Má elaboração dos impressos } \\
\text { •Necessidade de capacitações e } \\
\text { atualizações }\end{array}$ & \\
\hline A5 & $\begin{array}{lr}\text { Sistematização } & \text { da } \\
\text { assistência } & \text { de } \\
\text { enfermagem: } & \\
\text { percepção } & \text { e } \\
\text { conhecimento } & \text { da } \\
\text { enfermagem } & \\
\text { Brasileira } & \end{array}$ & $\begin{array}{l}\text { 2019/ MEDLINE/ } \\
\text { Revista Brasileira de } \\
\text { Enfermagem }\end{array}$ & \begin{tabular}{lr} 
Marcos & Renato de \\
Oliveira; Paulo César de \\
Almeida; Thereza Maria \\
Magalhães & Moreira; \\
Raimundo & Augusto \\
\multicolumn{2}{l}{ Martins Torres }
\end{tabular} & Descritivo & $\begin{array}{l}\text { - Divergência entre raciocínio e } \\
\text { prática } \\
\text { - Falta de conhecimento teórico. } \\
\text {-A percepção de que o PE é } \\
\text { irrelevante. } \\
\text {-A utilização teve associação } \\
\text { estatística com maior nível de } \\
\text { formação. }\end{array}$ & VI \\
\hline A6 & $\begin{array}{lr}\text { Sistematização } & \text { da } \\
\text { assistência } & \text { de } \\
\text { enfermagem: } & \\
\text { vantagens } & \text { e } \\
\text { dificuldades na sua } \\
\text { aplicação sob a a } \\
\text { ótica de enfermeiros }\end{array}$ & $\begin{array}{lr}2019 / & \text { BDENF/ } \\
\text { Revista } & \text { Eletrônica } \\
\text { Acervo Saúde / } & \text { Saurdronic Journal } \\
\text { Electron } & \text { Collection Health }\end{array}$ & 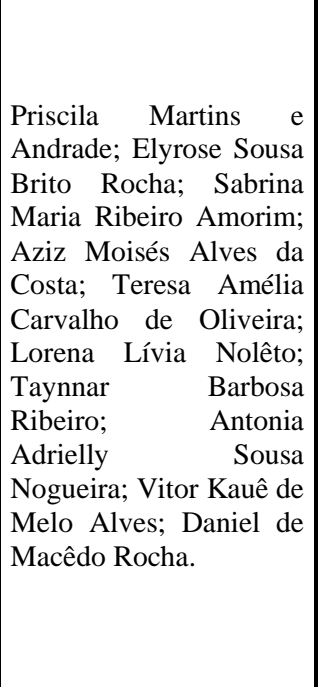 & Descritivo & $\begin{array}{l}\text { •Desinteresse, resistência e } \\
\text { desvalorização do método } \\
\text { - Falta de conhecimento prático } \\
\text { - Falta de conhecimento teórico } \\
\text { - Número reduzido de profissionais } \\
\text { - Sobrecarga de trabalho } \\
\text { - Necessidade de um maior amparo e } \\
\text { envolvimento da gerência, da } \\
\text { instituição e dos órgãos responsáveis } \\
\text { pelos profissionais de enfermagem. } \\
\text { - Falta de tempo } \\
\text { - Falta de continuidade do PE e SAE } \\
\text { pela equipe }\end{array}$ & VI \\
\hline A7 & 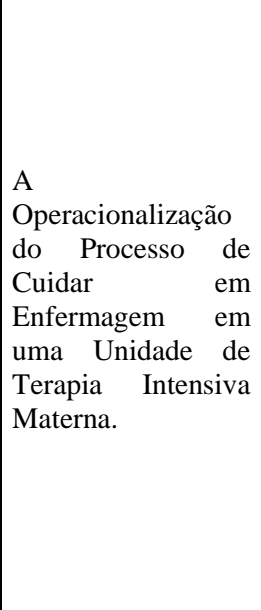 & $\begin{array}{l}\text { 2018/BDENF/ } \\
\text { Revista Online de } \\
\text { Pesquisa Cuidado é } \\
\text { Fundamental }\end{array}$ & $\begin{array}{lrr}\text { Magda } & \text { Rogéria P. } \\
\text { Viana; Indyara } & \text { Maria } \\
\text { Barros } & \text { Silva; } & \text { Thamires } \\
\text { Raquel } & \text { Silva } & \text { Ferreira; } \\
\text { Fernanda } & \text { Claúdia } \\
\text { Miranda Amorim; Érida } \\
\text { de Oliveira Soares. }\end{array}$ & Descritivo & $\begin{array}{l}\text {-Desinteresse, resistência e } \\
\text { desvalorização do método } \\
\text { - Falta de conhecimento prático } \\
\text { - Falta de conhecimento teórico } \\
\text { - Número reduzido de profissionais } \\
\text {-Processo de trabalho } \\
\text { individualizado/falha no trabalho } \\
\text { - Sobrecarga de trabalho } \\
\text {-Necessidade de capacitações e } \\
\text { atualizações }\end{array}$ & VI \\
\hline A8 & $\begin{array}{ll}\text { Sistematização } & \text { da } \\
\text { Assistência } & \text { de } \\
\text { Enfermagem: } & \\
\text { percepção } & \text { dos } \\
\text { enfermeiros. } & \end{array}$ & $\begin{array}{l}\text { 2018/BDENF/ } \\
\text { Revista Online de } \\
\text { Pesquisa Cuidado é } \\
\text { Fundamental }\end{array}$ & $\begin{array}{l}\text { Denise Consuelo Moser; } \\
\text { Gelson Aguiar da Silva; } \\
\text { Suellen Rodrigues de } \\
\text { Oliveira; Leonardo } \\
\text { Costa Barbosa; Tatiana } \\
\text { Gaffuri da Silva. }\end{array}$ & Descritivo & $\begin{array}{l}\text { - Afastamento do raciocínio crítico e } \\
\text { o julgamento da enfermagem } \\
\text { - Desconhecimento em relação ao PE } \\
\text { - Divergência entre raciocínio e } \\
\text { prática } \\
\text {-Empirismo nas atividades } \\
\text { desenvolvidas } \\
\text { - Falta de conhecimento teórico } \\
\text {-Número reduzido de profissionais }\end{array}$ & VI \\
\hline
\end{tabular}




\begin{tabular}{|c|c|c|c|c|c|c|}
\hline & & & & & $\begin{array}{l}\text {-Sobrecarga de trabalho } \\
\text { •Necessidade de capacitações e } \\
\text { atualizações }\end{array}$ & \\
\hline A9 & $\begin{array}{l}\text { Construção de um } \\
\text { software com o com } \\
\text { o Processo de } \\
\text { Enfermagem em } \\
\text { Terapia Intensiva. }\end{array}$ & $\begin{array}{l}\text { 2016/LILACS/ } \\
\text { Journal of Health } \\
\text { Informatics }\end{array}$ & $\begin{array}{l}\text { Meire Chucre Tannure } \\
\text { Martins; Tânia Couto } \\
\text { Machado Chianca. }\end{array}$ & Descritivo & $\begin{array}{l}\text { - Falta de conhecimento prático } \\
\text {-Falta de instrumentos elaborados e } \\
\text { implementados previamente } \\
\text { - Manuseio do sistema informatizado }\end{array}$ & VI \\
\hline A10 & $\begin{array}{l}\text { Processo de } \\
\text { enfermagem } \\
\text { informatizado em } \\
\text { Unidade de terapia } \\
\text { intensiva: } \\
\text { ergonomia } \\
\text { usabilidade. }\end{array}$ & $\begin{array}{l}\text { 2016/LILACS/ } \\
\text { Revista da Escola de } \\
\text { Enfermagem da } \\
\text { USP }\end{array}$ & $\begin{array}{l}\text { Sônia Regina Wagner de } \\
\text { Almeida; } r \text { Grace } \\
\text { Teresinha Marcon Dal } \\
\text { Sasso; Daniela Couto } \\
\text { Carvalho Barra. }\end{array}$ & $\begin{array}{l}\text { Semi- } \\
\text { experiment } \\
\text { al }\end{array}$ & $\begin{array}{l}\text { - Má elaboração dos impressos } \\
\text { - Volume de informações exacerbado } \\
\text { na elaboração do PE } \\
\text { - Necessidade de capacitações e } \\
\text { atualizações } \\
\text { - Manuseio do sistema informatizado }\end{array}$ & IV \\
\hline
\end{tabular}

Fonte: Elaboração própria.

A partir dos resultados encontrados e com o objetivo de melhorar a descrição e o entendimento dos estudos, foram elencadas duas categorias temáticas, conforme a seguir: "Dificuldades vivenciadas por enfermeiros no processo de sistematização da assistência na UTI" e "Desafios relacionados à estruturação do processo na implementação da SAE nas UTIs".

\subsection{Dificuldades Vivenciadas por Enfermeiros no Processo de Sistematização da Assistência na UTI}

Nesta categoria temática, serão pleiteadas as dificuldades vivenciadas por enfermeiros na implementação do processo de sistematização da assistência nas Unidades de Terapia Intensiva. Dessa forma, os desafios encontrados foram: desconhecimento em relação ao PE e a SAE; falta de conhecimento teórico; falta de conhecimento prático; afastamento do raciocínio crítico e o julgamento da enfermagem; divergência entre raciocínio e prática, empirismo nas atividades desenvolvidas, número reduzido de profissionais; sobrecarga de trabalho, falta de tempo, falta de continuidade pela equipe, desinteresse, resistência e desvalorização do método e volume de informações exacerbado na elaboração do PE.

Dentre os problemas, o mais citado foi a falta de conhecimento teórico. Oito artigos (A2, A3, A4, A5, A6, A7, A8 e A10) o apontaram como principal desafio para a implementação da SAE na UTI.

De acordo com a pesquisa realizada por Viana, et al. (2018) observou-se que a realidade enfrentada pelos enfermeiros em relação à operacionalização deste processo é insuficiente em algumas etapas no sentido do cumprimento das etapas do mesmo devido ao conhecimento fragilizado dos enfermeiros, percebeu-se que as ações do enfermeiro são realizadas, muitas vezes, desvinculadas da SAE, o que dificulta a singularidade do indivíduo para o atendimento de suas particularidades.

Por tratar-se de uma UTI, espera-se um nível maior de dependência por parte dos pacientes. Entretanto, sabemos que a UTI comporta uma infraestrutura especializada, na qual a prestação de cuidados aos pacientes críticos requer do enfermeiro o domínio de várias tecnologias, a fim de ser realizado um cuidado especializado. Todavia, não foi observada, durante a realização da pesquisa, a avaliação efetiva das prescrições definidas, e ressalta-se ainda um afastamento do raciocínio crítico e o julgamento de Enfermagem pelos enfermeiros (Moser, et al., 2018).

Segundo ao estudo de Oliveira, et al. (2019), percebe-se que o nível de conhecimento da Enfermagem brasileira sobre SAE varia proporcionalmente, conforme o nível educacional do indivíduo e por ter recebido formação sobre SAE na graduação. Acreditar que a SAE é irrelevante, associou-se com não receber nem buscar formação extra sobre o tema e acreditar que raciocínio clínico e teorias de enfermagem são pouco relevantes. Assim, denota-se que são os fatores de percepção e 
conhecimento que influenciam na aplicação da SAE na prática clínica, pois a percepção da relevância e o nível de conhecimento são diretamente relacionados com o uso/não uso da SAE.

O estudo realizado por Andrade, et al. (2019) em relação às dificuldades encontradas pelos enfermeiros para realizar a SAE, apontou a pouca quantidade de profissionais, resultando em uma sobrecarga de trabalho. Mencionou ainda a falta de tempo para realizar suas funções de forma plena. Sabe-se que em uma clínica o enfermeiro exerce tanto a assistência quanto a gerência do serviço, e um ponto citado é o fato do profissional se dividir entre o cuidado com o paciente e a burocracia que o serviço demanda.

A ausência da realização do exame físico/entrevista pelo enfermeiro dificulta a abordagem adequada dos problemas rentados pelo paciente e a avaliação dos resultados obtidos com as intervenções prescritas/realizadas. A falta do registro dessa etapa dificulta as avaliações subsequentes, a permanência ou exclusão de diagnósticos, alterações da prescrição e evolução de enfermagem. As médias do número de fases documentadas por tipo de setor mostram que as UTI são os setores que mais se aproximaram das cinco categorias de documentação (Azevedo, et al., 2019).

Segundo a pesquisa realizada por Andrade, et al. (2019), os enfermeiros entrevistados afirmaram que a SAE contribui para a melhoria da assistência da equipe de enfermagem, ao passo que direciona e permite a continuidade do cuidado, como também organiza o serviço.

Dessa maneira, o enfermeiro sente-se mais seguro durante a prestação dos cuidados, pois a SAE também permite uma conduta mais fundamentada, respaldada em conhecimento científico; assim sendo, o profissional deixa de atuar de forma intuitiva ou impulsiva, só cumprindo tarefas, como, na maioria das vezes, ele é visto. Com a SAE, ele ganha autonomia para as tomadas de decisões, além de serem embasadas e planejadas, construindo seu próprio conhecimento e contribuindo cada vez mais para consolidar a enfermagem como ciência (Costa \& Silva, 2020).

Dessa forma, a obrigatoriedade legal em relação ao uso da SAE em qualquer instituição de saúde infelizmente não é aplicada em sua totalidade. São muitos desafios enfrentados pelos enfermeiros que estão propícios a condições trabalhistas escassa, o que acaba influenciando na implementação eficaz da SAE nas Unidades de Terapia Intensiva.

\subsection{Desafios relacionados à estruturação do processo na implantação da SAE nas UTIS}

Nesta categoria temática, serão discutidos os desafios relacionados à estruturação do processo nas UTIs.

Os desafios em relação à estrutura foram encontrados em seis artigos (A1, A2, A3, A6, A7 e A9) sendo eles: falta de instrumentos elaborados e implementados previamente; instrumentos de registro do PE não contemplam a complexidade dos cuidados específicos; má elaboração dos impressos e volume de informações exacerbado na elaboração do PE, manuseio do sistema informatizado e processo de trabalho individualizado/falha no trabalho.

De acordo com Azevedo, et al. (2019) os enfermeiros não reconhecem que têm um papel clínico junto aos usuários dos serviços de saúde, que suas ações de cuidado influenciam a saúde das pessoas, não haverá preparo que consiga diminuir a resistência ao uso do PE ou políticas institucionais capazes de incorporar modelos de assistência compatíveis com o uso do PE. A ausência de registro ou o registro inadequado pode resultar em: descontinuidade da assistência, avaliação inadequada das alterações das condições clínicas do paciente, julgamento impreciso dos resultados obtidos, ausência de base jurídica consistente para defesa quanto ao trabalho realizado ou quanto ao cuidado recebido.

O enfermeiro como responsável pela equipe de enfermagem tem responsabilidade na operacionalização do processo de cuidar, pois ele deve ser o grande incentivador da equipe para que o PE seja executado em sua totalidade. Para que isso seja possível, o líder da equipe deve saber o que é o PE, como ele deve ser operacionalizado e sua importância para a assistência associado à vontade de mudar a realidade atual (Viana, et al., 2018). 
Segundo a pesquisa de Santos, et al. (2020) os discursos dos profissionais revelaram o histórico de enfermagem, como uma tecnologia do cuidado que orienta o raciocínio, melhora a qualidade do cuidado por meio da sistematização da avaliação clínica, com ênfase no fortalecimento da autonomia e empoderamento profissional. Sua utilização, bem como das demais etapas do PE, representam o avanço da enfermagem frente às demandas impostas no dia a dia dos serviços de saúde, bem como, uma das características de boas práticas em enfermagem.

De acordo com Martins \& Chianca (2016) as taxas de efetividade servem para ser o alicerce do banco de dados, utilizar linguagens padronizadas para alimentar o sistema e incorporar recursos capazes de permitir o acesso à informação, o cruzamento de dados, a individualização do cuidado, a tomada de decisão e a obtenção de indicadores para a avaliação da assistência prestada aos pacientes.

No estudo de Almeida, et al. (2016) a usabilidade do sistema informatizado foi avaliada como "excelente" pelos enfermeiros, professores e programadores. Concluiu-se que esta tecnologia integra uma lógica de dados, informações, avaliação clínica, diagnósticos e intervenções de enfermagem possibilitando aos enfermeiros a sua utilização em Unidades de Terapia Intensiva, por possuir conteúdo completo e atualizado e estar alicerçada na Classificação Internacional para as Práticas de Enfermagem versão 1.0 .

Conforme Andrade, et al. (2019), sobre as contribuições da SAE para a melhoria do cuidado, os enfermeiros relataram que a sistematização favorece a assistência, pois é uma forma de respaldo para o profissional de enfermagem à medida que este registra todo e qualquer procedimento realizado. Bem como imprime autonomia à classe de enfermeiros, pois dá maior visibilidade para a profissão, referiram também que a SAE melhora a comunicação entre a equipe, conforme são feitas as anotações no prontuário do paciente. Mencionaram ainda que a sistematização aproxima não só os integrantes da equipe em si, mas também aproxima o profissional do cliente.

Segundo o estudo de Silva, et al. (2021) os enfermeiros perceberam que a implementação e aplicação do PE na UTI permitiu a melhora da qualidade da assistência, o conhecimento sobre o paciente foi aprofundado. Destaca-se que o uso da estratégia do grupo de estudos adotada pelos enfermeiros durante a implementação do PE, se mostrou como positiva com potencial para utilização em novos contextos e com maior detalhamento em estudos futuros. As reflexões constituem um corpo de conhecimento relevante para a assistência em enfermagem ao evidenciar as melhorias obtidas na assistência após a implementação do Processo de Enfermagem, por meio de uma assistência baseada cientificamente, assim como, o reconhecimento profissional por meio de registros adequados.

Apesar de essas inúmeras dificuldades encontradas para implementação da SAE, cabe aos enfermeiros e às instituições responsáveis um maior envolvimento, capacitação profissional e ética aliado ao conhecimento científico para buscar melhorias e valorização profissional e dos impressos do PE.

\section{Considerações Finais}

A análise dos estudos selecionados permitiu perceber que os enfermeiros reconhecem a SAE e o PE como metodologias que organizam a prática de Enfermagem. O principal desafio exposto foi o déficit de conhecimento teórico, prático e clínico. Além disso, o número reduzido de profissionais, sobrecarga de trabalho, falta de continuidade pela equipe, resistência e desvalorização do método, falta de instrumentos elaborados e implementados previamente, má elaboração dos impressos e manuseio do sistema informatizado foram os outros desafios mais citados nos estudos. Destacou-se também que os profissionais de enfermagem necessitam ter como complemento para seus conhecimentos a educação permanente, juntamente com a educação continuada devido à sua importância da SAE e do PE.

Dessa forma, a introdução de disciplinas que englobem a SAE nas graduações de enfermagem, ministradas por docentes capacitados, bem como, o maior envolvimento da instituição hospitalar e investimento na capacitação dos enfermeiros da UTI, 
contribuem para a conquista de benefícios próprios para a instituição e para os pacientes, por meio de assistência de enfermagem competente, resolutiva, focada e qualificada ao cuidado crítico. No entanto, são necessários mais estudos que demonstrem a importância de implementação da SAE.

\section{Referências}

Abreu, A. J. P (2020). Desafios da equipe de enfermagem para a implementação do processo de enfermagem em unidades de terapia intensiva: revisão integrativa [dissertação]. Centro Universitário Fametro - UNIFAMETRO.

Almeida, S., et al. (2016). Computerized nursing process in the Intensive Care Unit: ergonomics and usability. Rev Esc Enferm USP. 50(6):996-1002.

Andrade, P. M et al.(2019). Sistematização da assistência de enfermagem: vantagens e dificuldades na sua aplicação sob a ótica de enfermeiros. Revista Eletrônica Acervo Saúde, 11(8), e588.

Azevedo, O. A et al (2019). Documentation of the nursing process in public health institutions. Rev Esc Enferm USP. 53 :e03471.

Brasil. Ministério da Saúde (2016). Conselho Nacional de Saúde. Resolução n. 510, de 07 de abril de 2016. Aprova diretrizes e normas regulamentadoras de pesquisas envolvendo seres humanos. Brasília, Diário Oficial da União.

Costa, A. C., \& Silva, J. V (2020). Significados de sistematização da assistência de enfermagem sob a ótica de enfermeiros. Revista Práxis. 12(23).

Gutierres, L. S et al (2018). Good practices for patient safety in the operating room: nurses' recommendations. Rev Bras Enferm 71(6).

Martins, M. C. T., \& Chianca, T. C. M. (2016). Construção de um software com o com o Processo de Enfermagem em Terapia Intensiva. J. Health Inform. outubro -dezembro; 8(4):119-125

Mendes, K. D. S \& Silveira, R. C. C. P., \& Galvão, C. M (2008). Revisão integrativa: método de pesquisa para a incorporação de evidências na saúde e na enfermagem. Texto Contexto Enferm, 14(4), 758 - 764.

Moser, D. C, et. al. (2018. Sistematização da Assistência de Enfermagem: percepção dos enfermeiros. Rev Fun Care Online. V. 10(4):998-1007.

Nery, I. S. \& Santos, A. G.\& Sampaio, M. R. F. B (2013). Dificuldades para a implantação Sistematização da Assistência de Enfermagem em maternidades. Enfermagem em Foco. 4(1), 11-14.

Neto, J. P \& Schulze, C. J (2015). Direito à saúde: análise à luz da judicialização. Verbo Jurídico. https://www.lexml.gov.br/urn/urn:le x:br:rede.virtual.bibliotecas:livro:2015;001051541.

Nunes, R. M, et. al (2019). Sistematização da assistência de enfermagem e os desafios para sua implantação na unidade de terapia intensiva: uma revisão de literatura. Rev UNINGÁ. 56(S2):80-93.

Oliveira, M. R, et. al (2019). Nursing care systematization: perceptions and knowledge of the Brazilian nursing. Rev Bras Enferm. 72(6):1547-53.

Santos, M. G, et. al. (2020). Boas Práticas de Enfermagem na Unidade de Terapia Intensiva: Desenvolvimento o histórico de enfermagem. Enferm Foco. 10, 193-201.

Silva, A. M, et. al. (2021). Percepções dos enfermeiros acerca da implementação do processo de enfermagem em uma unidade intensiva Rev Gaúcha Enferm.

Silva, M. C. N.(2017) Sistematização da assistência de Enfermagem: desafio para a prática profissional. Enfermagem em Foco. 8 (3).

Swiger, P. A, et al. (2016). Nursing workload in the acute-care setting: a concept analysis of nursing workload. Nurs Outlook. 64(3):244-54;

Ursi, E. S. \& Gavão C. M (2006). Prevenção de lesões de pele no perioperatório: revisão integrativa da literatura. Rev. Latino-Am. Enfermagem [online]. 14(1), $124-131$.

Viana, M. R. P, et al.(2018). A Operacionalização do Processo de Cuidar em Enfermagem em uma Unidade de Terapia Intensiva Materna. Rev Fund Care Online. 10(3):696-703. 